\title{
CRECIMIENTO, PRODUCCIÓN Y CALIDAD DE FLORES EN CALAS (Zantedeschia aethiopica (L.) K. Spreng) EXPUESTAS A DIFERENTE CALIDAD DE LUZ
}

\section{GROWTH, PRODUCTION AND FLOWER QUALITY IN CALLA LILY (Zantedeschia aethiopica (L.) K. Spreng) EXPOSED TO DIFFERENT LIGHT QUALITY}

\author{
Fánor Casierra-Posada1, Paola J. Nieto², Christian Ulrichs³
}

\begin{abstract}
${ }^{1}$ Ingeniero Agrónomo, PhD. Facultad de Ciencias Agropecuarias, Universidad Pedagógica y Tecnológica de Colombia UPTC, Tunja. Grupo de investigación Ecofisiología Vegetal. E-mail: fanor.casierra@uptc.edu.co (Autor para correspondencia). 2 Ingeniera Agrónoma, Universidad Pedagógica y Tecnológica de Colombia - UPTC. ${ }^{3}$ Prof. Dr. Dr. Humboldt-Universität zu Berlin, Landwirtschaftlich-Gärtnerische Fakultät, Fachgebiet Urbane Ökophysiologie der Pflanzen, Lentzeallee 55/57, 14195 Berlin, Germany.
\end{abstract}

Rev. U.D.C.A Act. \& Div. Cient. 15(1): 97 - 105, 2012

\section{RESUMEN}

La cantidad, la calidad y la duración de la luz afectan el crecimiento vegetal. La calidad de la luz, se refiere al color o la longitud de onda que llega a la planta. La manipulación espectral, se orienta a promover, específicamente, respuestas fisiológicas deseadas. Se estudió la influencia de la calidad de la luz sobre el crecimiento, la producción y la calidad de flor en calas (Zantedeschia aethiopica). Las plantas, se cultivaron en el municipio de Tunja, Colombia, en invernadero, bajo diferentes calidades de luz, proporcionadas por películas de polipropileno (control sin cobertura de color, amarillo, verde, azul transparente y rojo). Las películas de color permitieron, también, diferentes niveles de sombreado. Los tratamientos, se dispusieron en un diseño de bloques completos al azar, con diez repeticiones. Las películas del filtro, se colocaron $1 \mathrm{~m}$ por encima del cultivo, desde el momento del trasplante hasta la cosecha de las plantas. La calidad de la flor, en cuanto a la longitud del pedúnculo, fue afectada positivamente por el sombreado; sin embargo, el número de flores, su tiempo de aparición y la asignación de masa seca a las flores no fueron influenciados por los tratamientos aplicados. Las respuestas de las plantas de cala en relación con las tasas de crecimiento fueron la consecuencia de la acción conjunta de la cantidad y la calidad de la luz incidente. Cala mostró gran versatilidad para adaptarse a las condiciones de la oferta ambiental, lo que se manifestó en modificaciones morfoanatómicas y fisiológicas en los vegetales, que crecieron bajo condiciones de espectro manipulado.
Palabras clave: Zantedeschia aethiopica, área foliar, masa seca, fotomorfogénesis, flores de corte.

\section{SUMMARY}

The quantity, quality and duration of light affect plant growth. Light quality refers to color, or wavelength, that reaches the plant. The spectral manipulation is aimed to specifically promote desired physiological responses. The influence of light quality on growth, production and flowers quality was studied in calla lilly plants (Zantedeschia aethiopica). Plants were grown in Tunja / Colobia, in a greenhouse, under different light qualities (control without colored cover, yellow, green, blue transparent and red) provided by polypropylene filter film. The colored films provided too different levels of shading to plants. The treatments were laid out in a randomized complete block design where each treatment was replicated ten times. The filter films were set $1 \mathrm{~m}$ above the crop canopy at transplanting until the harvest of plants. The flowers quality related to the length of peduncle was positively affected by shading, however the number of flowers, the flowering time and the dry mass allocation to flowers were not influenced by the applied treatments. The response of plants in relation to growth rates were the result of joint action of both, the quantity and quality of incident light. Calla lily showed versatility to adapt to environmental conditions, which was manifested through physiological and morpho-anatomical changes in plants growing under manipulated spectra. 
Key words: Zantedeschia aethiopica, leaf area, dry mass, photomorphogenesis, cut flowers.

\section{INTRODUCCIÓN}

Zantedeschia sp. es una planta de origen sudafricano (CruzCastillo et al. 2008), conocida como cala, lirio de agua o cartucho; es una especie bulbosa, cuyo cultivo mundial es relativamente reciente (Funnell, 1994). Entre los factores que limitan el incremento de las áreas sembradas en Colombia y en otros países, se encuentran la falta de información sobre el manejo agronómico, los altos costos de material vegetal y la susceptibilidad de los bulbos al ataque de agentes patógenos.

Esta planta es apreciada por sus inflorescencias, que consisten de numerosas flores adjuntas a lo largo de un espádice envuelto por una espata, la cual, junto con el espádice, sostenidos por el pedúnculo, se consideran como la flor (Funnell, 1993). Generalmente, su producción protegida y a cielo abierto ocurre en áreas con clima templado, como en Estados Unidos de Norteamérica, en Nueva Zelanda, en Italia y en México (Cruz-Castillo et al. 2008). En Colombia, se le cultiva tanto en invernadero como a cielo abierto. Estas plantas presentan fotoperiodo neutro (Robinson et al. 2000) y prefieren temperaturas entre 15 y $28^{\circ} \mathrm{C}$ que, por lo general, son proporcionadas dentro de invernaderos (De Pascale \& Paradiso, 2006)

Por otra parte, la luz, uno de los factores más importantes del entorno vegetal, no sólo es un sustrato energético, sino un regulador de procesos fisiológicos en los vegetales (Alyabyev et al. 2002). Se han determinado utilizando plantas de Arabidopsis, tres tipos de receptores de la luz: el criptocromo 1 , el criptocromo 2 y la fototropina, los cuales, regulan, primordialmente, la inhibición del hipocótilo, el momento de la floración y el fototropismo, respectivamente (Lin, 2000). Se han realizado ensayos para evaluar la respuesta de las plantas a la luz de diferentes colores. Es así, como se encontró que la regulación de la posición de las hojas de Arabidopsis sp. es altamente dependiente de la luz azul y roja (Inoue et al. 2008). En plantas de fresa, en relación con las plantas que crecieron a libre exposición, los valores de la fluorescencia $F_{v}, F_{m}$ y $F_{t}$ fueron mayores en las plantas colocadas bajo coberturas de polipropileno de colores amarillo, verde, azul, rojo y transparente; además, el valor de $F_{0}$ fue mayor bajo las coberturas amarilla y transparente, mientras que el cociente $F_{v} / F_{m}$ fue menor bajo las películas de estos colores (Casierra-Posada et al. 2011)

Dado que se deben presentar a los cultivadores alternativas de cultivo que incrementen el rendimiento, el objetivo del presente estudio fue la evaluación del efecto de cubrimiento de diferentes colores sobre los parámetros básicos del crecimiento, la producción y la calidad de la flor en plantas de cala, cultivadas bajo invernadero.

\section{MATERIALES Y MÉTODOS}

El ensayo, se llevó a cabo en el municipio de Tunja, Colombia, en la Universidad Pedagógica y Tecnológica de Colombia UPTC, bajo condiciones de invernadero. En promedio, se registró dentro del invernadero, una temperatura de $16,2^{\circ} \mathrm{C}$ y $71,8 \%$ de humedad relativa, durante el ensayo. Se utilizaron plantas de cala, cuya propagación se realizó de manera vegetativa. El material usado para las coberturas fue papel celofán de colores: transparente, azul, amarillo, rojo y verde. Las características de radiación y de opacidad registradas bajo las coberturas, se presentan en la tabla 1 , las cuales, fueron determinadas con un medidor de luz Fieldscout 3415F (Spectrum Technologies, Inc, USA). Las plantas control crecieron bajo las mismas condiciones que las demás, pero sin cobertura. Se adecuó el invernadero con tubería y con mangueras, para la conexión de un sistema de aireación a contenedores de vidrio, con el fin de oxigenar las plantas. Los contenedores, se cubrieron con papel aluminio y se colocaron las plántulas en una solución nutritiva completa. Se montaron bastidores forrados cada uno, con una cobertura diferente de color.

La solución nutritiva $(\mathrm{pH} 6,5)$ tenía la siguiente composición en $\mathrm{mg} \mathrm{L}^{-1}$ : nitrógeno nítrico, 40,3; nitrógeno amoniacal, 4,0; fósforo, 20,4; potasio, 50,6; calcio, 28,8; magnesio, 11,4; azufre, 1,0; hierro, 1,12; manganeso, 0,112; cobre, 0,012; zinc, 0,0264; boro, 0,106; molibdeno, 0,0012 y cobalto, 0,00036 . Los bastidores, se colocaron sobre las mesas, cada uno con diez plántulas en su interior, separadas $40 \mathrm{~cm}$.

Se realizó el seguimiento del consumo de agua, que se tomaba una vez por semana, como el agua faltante en los contenedores. Con este valor, se calculó la eficiencia en el uso del agua, como el cociente entre la masa seca total por planta y el agua consumida, durante el tiempo del ensayo. De la misma manera, se determinó el tiempo de aparición de la flor, mediante monitoreos semanales. Se evaluó la calidad de la flor dada por la longitud del tallo y el diámetro de la base de la espata. Para la toma de esta variable, se realizaba el corte en la base de la flor para luego ser medida con un metro y con un pie de rey el diámetro; así mismo, se llevaba el registro del número de flores cortadas en cada planta, según los tratamientos.

Para la determinación de la masa seca, se tomó cada planta y se separó la raíz de los pecíolos y éstos de las hojas, a las cuales, se les hacia la lectura de área foliar, con un medidor Li-cor 3000A (Li-cor, Lincoln, Nebraska - USA). 
Tabla 1. Radiación y opacidad medidas bajo las coberturas de color.

\begin{tabular}{|l|c|c|}
\hline \multirow{2}{*}{ Color de la cobertura } & Radiación fotosintéticamente activa & Opacidad \\
\cline { 2 - 3 } & $\left(\mu \mathrm{mol} \mathrm{m}^{-2} \mathrm{~s}^{-1}\right)$ & $(\%)$ \\
\hline Control & 299,94 & 0 \\
\hline Transparente & 210,46 & 29,83 \\
\hline Azul & 99,31 & 66,89 \\
\hline Amarillo & 152,19 & 49,25 \\
\hline Rojo & 86,95 & 71,01 \\
\hline Verde & 78,87 & 73,70 \\
\hline
\end{tabular}

Al inicio del experimento, se registró el peso seco de los diferentes órganos en diez plantas de las plántulas, tomadas como material de siembra, con miras a tener estos valores iniciales, para el cálculo de los índices de crecimiento, según la metodología reportada por Hunt (1990).

Se utilizaron 60 plantas, 6 tratamientos, cada uno con 10 plantas. Cada tratamiento correspondió a un color de cobertura (control sin cobertura de color, amarillo, verde, azul transparente y rojo). Para el análisis, se tomó una planta, como unidad experimental; se dispuso de un diseño completamente al azar, con 10 replicaciones. Se realizó el análisis de varianza (ANAVA) y los tratamientos se compararon, mediante la prueba de separación de promedios de Tukey, con un nivel de significancia del $5 \%$. Los análisis estadísticos, se realizaron con la versión 17.0.0 de SPSS (Statistical Product and Service Solutions, Chicago, Illinois, USA).

\section{RESULTADOS Y DISCUSIÓN}

Consumo de agua: Con relación a las plantas control sin cobertura, el consumo de agua se redujo en todas las plantas expuestas a las coberturas de color, incluso, en la cobertura transparente, con diferencias altamente significativas $(P<0,01)$. En comparación con las plantas control sin cobertura, la mayor reducción se presentó en las plantas que se desarrollaron bajo la cobertura azul, con $22,68 \%$ menos consumo de agua. Por su parte, la cobertura de color verde provocó una reducción del consumo de agua de $8,95 \%$ (Tabla 2).

De acuerdo con Maleszewski \& Kozłowska-Szerenos (1998), tanto la luz roja como la azul afectan la apertura estomática; sin embargo, algunos autores consideran que sólo la luz azul es la responsable de este mecanismo (Lawson et al. 2002). Rabiza-Świder \& Skutnik (2004) encontraron que en hojas cortadas de $Z$. aethiopica, la mayor apertura estomática
$(8,2 \mu \mathrm{m})$ se observó con la exposición a la luz azul. Bajo condiciones de luz roja, los estomas mantuvieron una apertura de $4,6 \mu \mathrm{m}$, lo que sería casi la mitad de la encontrada con la luz azul. Por tanto, sería de esperarse que en el presente estudio, las plantas expuestas a la luz azul tuvieran mayor consumo de agua que aquellas colocadas bajo la cobertura de color rojo; no obstante, sucedió lo contrario, puesto que al analizar el consumo de agua, sin excluir el efecto de la apertura de los estomas, se debe considerar también el área foliar. Fue así como las plantas bajo la cobertura roja tuvieron mayor área foliar que las que crecieron bajo la cobertura azul, lo que les permitió una mayor posibilidad de transpiración, como se muestra en la tabla 2.

Eficiencia en el uso del agua: Bajo la cobertura de color azul, las plantas presentaron un valor de la eficiencia en el uso del agua $12,65 \%$ menor que las plantas control, mientras que las demás coberturas revelaron valores porcentuales por encima de las plantas control. Los valores más elevados, se registraron con las coberturas transparente y verde, cuyos promedios fueron 23,41 y $19,36 \%$ superiores a los registrados por las plantas control (Tabla 2). Al respecto, se hallaron diferencias significativas $(P<0,01)$.

Se debe tomar en consideración que la eficiencia en el uso del agua es una medida de la efectividad de los estomas en maximizar la fotosíntesis, reduciendo la pérdida de agua por transpiración (Hatfield et al. 2001). Wang et al. (2009) reportaron que en Cucumis sativus, las plantas que crecieron expuestas a luz monocromática mostraron crecimiento y tasa de asimilación de $\mathrm{CO}_{2}$ reducidos en comparación con aquellas que crecieron bajo luz blanca. La reducción en estos parámetros fue más notoria en plantas expuestas a luz de color verde, amarilla y roja. Tomado en consideración estos resultados, se puede justificar el mejor comportamiento de las plantas que crecieron bajo la cobertura transparente, en comparación con otros colores de cobertura o con el testigo. De hecho, los bastidores que sostenían las coberturas 
Tabla 2. Parámetros implicados en el crecimiento en calas (Zantedeschia aethiopica L.) expuestas a coberturas de colores.

\begin{tabular}{|c|c|c|c|c|c|c|}
\hline $\begin{array}{l}\text { Color de la } \\
\text { cobertura }\end{array}$ & $\begin{array}{c}\text { Consumo } \\
\text { de agua }\end{array}$ & $\begin{array}{l}\text { Eficiencia en el } \\
\text { uso del agua }\end{array}$ & $\begin{array}{c}\text { Masa } \\
\text { seca total }\end{array}$ & Área foliar & $\begin{array}{l}\text { Longitud } \\
\text { de la flor }\end{array}$ & $\begin{array}{c}\text { Diámetro de la } \\
\text { base de la espata }\end{array}$ \\
\hline & (L) & $\left(g \mathrm{~L}^{-1}\right)$ & (g) & $\left(\mathrm{cm}^{2}\right)$ & $(\mathrm{cm})$ & $(\mathrm{cm})$ \\
\hline Control & 20,96 b & $1,81 \mathrm{ab}$ & 37,71 bc & $640,21 \mathrm{bc}$ & $47,40 \mathrm{a}$ & $0,94 \mathrm{~b}$ \\
\hline Transparente & $18,23 \mathrm{ab}$ & $2,24 \mathrm{c}$ & 40,81 bc & $505,60 \mathrm{ab}$ & 47,28 a & $0,90 \mathrm{~b}$ \\
\hline Azul & 15,97 a & $1,58 \mathrm{a}$ & 26,48 a & 350,50 a & $49,57 \mathrm{ab}$ & $0,73 \mathrm{a}$ \\
\hline Amarillo & $17,01 \mathrm{a}$ & $1,91 \mathrm{abc}$ & $32,37 \mathrm{ab}$ & 381,70 a & $54,08 \mathrm{~b}$ & $0,88 \mathrm{~b}$ \\
\hline Rojo & $18,44 \mathrm{a}$ & $1,95 \mathrm{abc}$ & $36,07 \mathrm{bc}$ & $490,21 \mathrm{ab}$ & $55,12 \mathrm{~b}$ & $0,89 \mathrm{~b}$ \\
\hline Verde & $19,08 a b$ & $2,16 \mathrm{bc}$ & $41,31 \mathrm{c}$ & $727,58 \mathrm{c}$ & $68,40 \mathrm{c}$ & $0,98 \mathrm{~b}$ \\
\hline C.V.* & 14,69 & 18,55 & 22,73 & 36,90 & 16,32 & 12,21 \\
\hline
\end{tabular}

plásticas estaban cerrados por todos los costados, excepto por la parte de abajo. Esto pudo limitar, notablemente, la transpiración, lo que se puede verificar con la información presentada en la tabla 2, en la cual, se observa que las plantas control sin cobertura presentaron mayor consumo de agua que aquellas colocadas bajo la cobertura transparente. Además, tomando en consideración que, según Wang et al. (2009) la luz blanca mejora la tasa de asimilación de $\mathrm{CO}_{2}$ y el $\Phi P S$ II, en comparación con la exposición a luz monocromática, se justifica la mayor eficiencia en el uso del agua, expuesto por las plantas que crecieron bajo la cobertura transparente, las cuales, estuvieron puestas a luz blanca y tuvieron una evapotranspiración menor que las plantas control.

Masa seca total: El masa seca total registrado en las plantas fue superior al registrado en las plantas control, bajo las coberturas verde y transparente, con valores de 9,53 y 8,23\%, respectivamente (Tabla 2). Por su parte, las coberturas de color azul, amarillo y rojo indujeron una reducción de 31,85 14,15 y $4,34 \%$, respectivamente, en relación con las plantas control sin cobertura. Al respecto, se encontraron diferencias significativas $(P<0,01)$.

Jordi et al. (1994) mencionan que sólo la luz roja tiene la capacidad de posponer los procesos degradativos en las hojas de Alstroemeria sp. De igual forma, Rabiza-Świder \& Skutnik (2004) reportaron que la respuesta a la calidad de la luz varió en Z. aethiopica y en Hosta. De hecho, la exposición a la luz roja duplicó la longevidad de hojas cortadas de $Z$. aethiopica, en comparación con hojas expuestas a la luz azul, mientras que en Hosta, la luz azul prolongó la vida en florero de las hojas. El efecto positivo en ambas especies estuvo relacionado con el retraso en la degradación de la clorofila, en cada caso con la exposición de las hojas a luz azul o roja. Por otra parte, Jordi et al. (1994) mencionan que en hojas cortadas de alstroemeria, la luz roja reduce las pérdidas de clorofila y prolonga la actividad fotosintética. El resultado encontrado en hojas de $Z$. aethiopica, se puede comparar con la reducción en el peso seco total, obtenido en el presente trabajo, cuando las plantas se expusieron a la cobertura de color azul, puesto que evidenciaron la menor producción de masa seca, debido, posiblemente, a que la luz azul tiene un efecto negativo en la degradación de la clorofila y la este pigmento es el fotorreceptor responsable de la primera etapa, en la transformación de la energía de la luz solar en energía química.

Área foliar: El análisis estadístico arrojó diferencias altamente significativas $(P<0,01)$. La cobertura de color verde estimuló el desarrollo de las hojas, cuya área fue $13,64 \%$ mayor que la de las plantas control sin cobertura. Por otro lado, las coberturas de colores transparente, azul, amarillo y rojo indujeron una reducción del área foliar, en términos de 21,02; 45,25; 40,37 y 23,42\%, respectivamente, en relación con las plantas sin cobertura (Tabla 2).

El incremento en el área foliar en las plantas expuestas a la cobertura de color verde, se explica con base en la característica generalizada en la plantas que toleran la 
sombra, incrementar su área foliar bajo esta condición (Valladares et al. 2004). Cuando una especie tiene la capacidad adaptativa para compensar la deficiencia de luz ocasionada por el sombrado, hay un incremento en el área foliar, con lo cual, se logra la máxima absorción de la luz incidente (Moraes Neto et al. 2000). Por tanto, en el presente estudio, las plantas colocadas bajo la cobertura de color verde incrementarían su área foliar, para lograr una mayor captura de luz; sin embargo, llama la atención que la cobertura de color rojo, a pesar de tener una opacidad muy similar a la de la cobertura color verde, no indujo un incremento del área foliar, tan notorio como la de color verde, lo que se puede explicar con el hecho que la radiación en el rango del rojo es más activa para la fotosíntesis, mientras que la actividad en el rango del verde es bastante débil, como manifiestan Lazo \& Ascencio (2010); por ende, las plantas colocadas bajo la cobertura de color verde necesitaban mayor área foliar, para compensar la poca efectividad de la luz para el proceso fotosintético, en este rango del espectro.

Calidad de la flor: Los tratamientos con las coberturas de color no afectaron significativamente el número de flores por planta ni la precocidad en la aparición de las flores, pero sí la longitud de los pedúnculos $(\mathrm{P}<0,01)$, en donde sobresale el tratamiento con cobertura verde, que presentó pedúnculos $44,30 \%$ más largos que las plantas sin cobertura (Tabla 2). De igual manera, las plantas expuestas a coberturas de color azul, amarillo y rojo mostraron pedúnculos 4,57; 14,09 y $16,28 \%$ más largos que las plantas control. En cuanto a la longitud de los pedúnculos, las flores de plantas a libre exposición y expuestas a las coberturas de color transparente y azul, se encontraban en la $1^{\text {a }}$ selección, según los patrones de clasificación utilizados por Schnettler et al. (2006), quienes ubican en esta categoría, las flores con tallos que tienen una longitud de $40-50 \mathrm{~cm}$ y, en $2^{\mathrm{a}}$ selección, las flores con tallos de $30-40 \mathrm{~cm}$. Las coberturas de color amarillo, rojo y verde indujeron la producción de tallos de una calidad superior a la $1^{\mathrm{a}}$ selección, como se observa en la tabla 2 .

La exposición de las plantas a las coberturas de color ocasionó una reducción en el diámetro de la base de la espata, cuya reducción máxima se evidenció con la cobertura de color azul, que alcanzó un diámetro 22,34\% menor que en plantas sin cobertura. Las coberturas de color transparente, amarillo, rojo y verde indicaron valores del diámetro de la base de la espata 4,25; 6,38; 5,31 y 4,25\% menores que las plantas control (Tabla 2).

Es un hecho que la calidad de la luz afecta la longitud de los pedúnculos, puesto que Heo et al. (2003) revelaron que la longitud de los pedúnculos de Cyclamen persicum eran más cortos cuando se exponían a la luz fluorescente que a la luz de color rojo; sin embargo, Wilfret et al. (1976) demostraron que el sombreado incrementa la longitud de los pedúnculos en plantas de pompón (C. morifolium Ramat.). De hecho, el pedúnculo de la flor terminal fue $52 \%$ más largo que en plantas que crecían a plena exposición, puesto que el sombreado causa etiolación de los pedúnculos, lo que permite su elongación. Este reporte concuerda con el resultado del presente trabajo, cuando las plantas se desarrollaron bajo las coberturas de color verde y rojo, que presentaron las flores de mayor longitud (Tabla 2), debido a pedúnculos más largos, lo que coincide con las coberturas de mayor grado de opacidad $(73,70$ y $71,01 \%$, respectivamente). Por otra parte, Funnell (1994) menciona que la floración en Zantedeschia sp. no depende del fotoperiodo; no obstante, resalta la importancia de la intensidad de la luz en este evento, puesto que la longitud del peciolo se incrementa cuando las plantas se exponen a niveles reducidos de luz. Bajo un sombreado del $50 \%$, en verano (PAR: $15 \mathrm{~mol} \mathrm{~m}^{-2}$ ), la longitud del pedúnculo se puede incrementar entre 13 y $87 \%$, en comparación con plantas a plena exposición. El mismo autor menciona que la longitud del pedúnculo bajo condiciones de sombreado depende, en gran medida, del material vegetal y de la temperatura, dado que el incremento en la longitud del pedúnculo ocurre cuando las plantas crecen expuestas a temperaturas bajas y sombra. También se ha reportado la inhibición total de la floración, como consecuencia de un sombreado, del 40 ó $50 \%$.

Fukuda et al. (2002) detectaron que al irradiar con luz azul la parte superior del pedúnculo de flores de geranio (Pelargonium zonale), se deprimió la elongación celular en esa zona. Ellos indicaron que al realizar esta irradiación con luz en el rango del rojo lejano, las células de la corteza y de la médula eran $20 \mu \mathrm{m}$ más largas que con la luz roja, mientras que en pedúnculos iluminados con luz azul, la longitud de las células permaneció inalterada. Este reporte confirma el resultado hallado en el presente estudio, en el cual, las plantas que crecían bajo la cobertura azul exteriorizaron un diámetro menor en la base de la espata o parte superior del pedúnculo, que aquellas que se desarrollaron bajo condiciones de cobertura de color rojo (Tabla 2).

Tasa de crecimiento absoluto (TCA): El color de la cobertura afectó la TCA con diferencias estadísticamente significativas $(\mathrm{P}<0,01)$. Con excepción de las coberturas de color transparente y verde, todos los demás colores de la cobertura indujeron valores de la TCA por debajo del valor que reflejaron las plantas control sin cobertura, en comparación con las plantas control. Al respecto, la cobertura de color azul motivó un valor de la TCA 39,24\% menor que el valor encontrado en las plantas control, con diferencias significativas entre estos dos tratamientos (Tabla 3). 
Tabla 3. Relaciones de crecimiento en calas (Zantedeschia aethiopica L.) expuestas a coberturas de colores.

\begin{tabular}{|l|c|c|c|}
\hline $\begin{array}{c}\text { Color de la } \\
\text { cobertura }\end{array}$ & $\begin{array}{c}\text { Tasa de crecimiento } \\
\text { absoluto }\end{array}$ & $\begin{array}{c}\text { Tasa de crecimiento } \\
\text { relativo }\end{array}$ & Tasa de asimilación neta \\
\hline Control & $\left(\mathrm{g} \mathrm{d}^{-1}\right)$ & $\left(\mathrm{g} \mathrm{g}^{-1} \mathrm{~d}-1\right)$ & $\left(\mathrm{g} \mathrm{m}^{-1} \mathrm{~d}^{-1}\right)$ \\
\hline Transparente & $0,1417 \mathrm{~b}$ & $0,0034 \mathrm{~b}$ & $1,8409 \mathrm{ab}$ \\
\hline Azul & $0,1527 \mathrm{~b}$ & $0,0035 \mathrm{~b}$ & $2,2660 \mathrm{~b}$ \\
\hline Amarillo & $0,0861 \mathrm{a}$ & $0,0026 \mathrm{a}$ & $2,6585 \mathrm{a}$ \\
\hline Rojo & $0,1155 \mathrm{ab}$ & $0,0031 \mathrm{~b}$ & $2,0496 \mathrm{ab}$ \\
\hline Verde & $0,1318 \mathrm{~b}$ & $0,0033 \mathrm{~b}$ & $1,8468 \mathrm{ab}$ \\
\hline C.V.* & 27,46 & $0,0036 \mathrm{~b}$ & 23,39 \\
\hline $\begin{array}{l}\text { En cada columna, letras diferentes indican diferencias significativas según la prueba de Tukey }(\mathrm{P}<0,05) \\
\text { * Coeficiente de variación de Pearson }\end{array}$ \\
\hline
\end{tabular}

De manera similar a lo encontrado en el presente trabajo en relación con el valor de la TCA en plantas colocadas bajo la cobertura azul, en un ensayo con semillas de brócoli que germinaron bajo coberturas de color amarillo, azul, naranja y transparente, se compararon con las plántulas que crecían a libre exposición. Durante la fase del semillero, las plántulas expuestas a cobertura azul mostraron baja acumulación de materia seca, en relación con los demás colores (CasierraPosada \& Rojas, 2009). Se debe tomar en consideración que las hojas absorben fotones en los rangos del azul y del rojo del espectro de la radiación fotosintéticamente activa (RFA), mientras que la absorción en el verde es más débil (Lazo \& Ascencio, 2010). A pesar de esto, la cobertura de color azul tuvo un efecto depresivo sobre el valor de la TCA, reflejado también en la producción total de masa seca (Tabla 3 ), mientras que la TCA en plantas expuestas a la cobertura de color verde, no mostró diferencias estadísticamente significativas con las plantas control sin cobertura. Esto sugiere que las plantas de $Z$. aethiopica no muestran un buen desempeño en cuanto a la producción de asimilados, cuando se exponen a la luz con una alta proporción en el rango del azul.

Tasa de crecimiento relativo (TCR): Esta variable mostró un comportamiento muy similar al registrado para la TCA. Se encontraron diferencias estadísticamente significativas entre el valor de la TCR presentada por las plantas expuestas a la cobertura de color azul y los demás tratamientos, incluyendo las plantas control sin cobertura $(P<0,01)$. De igual modo, como se evidenció en la TCA, el valor de la TCR fue menor en las plantas expuestas a las coberturas de color azul, amarillo y rojo, mientras que las coberturas de color transparente y verde mostraron valores de TCA 3,62 y 4,58\%, por encima del valor registrado por las plantas control, sin diferencia significativa entre estos tres tratamientos. La cobertura de color azul indujo un valor de la TCR 22,01\% menor que las plantas sin cobertura, con diferencia estadísticamente significativa entre estos tratamientos (Tabla 3).

En relación con el comportamiento de los parámetros de crecimiento hallados en el presente ensayo, vale la pena mencionar que Wang et al. (2009), al exponer plantas de C. sativus a luz de diferentes colores, apreciaron que todas las plantas que crecieron expuestas a luz monocromática reflejaron tasa de asimilación de $\mathrm{CO}_{2}$ y rendimiento cuántico del transporte de electrones en el PSII (ФPS II), reducidos en comparación con aquellas que crecieron bajo luz blanca. La reducción en estos parámetros fue más notoria en plantas expuestas a luz de color verde, amarilla y roja. Además, la disminución en el valor del TPS II, se debió, principalmente, a la reducción en la disipación fotoquímica (photochemical quenching - qP).

Tasa de asimilación neta (TAN): Se encontró diferencia estadísticamente significativa en cuanto a este parámetro, en las plantas expuestas a las coberturas transparente y de color azul $(\mathrm{P}<0,01)$. A pesar que no se evidenció diferencia significativa para el valor de la TAN entre las plantas sin cobertura y aquellas expuestas a cobertura transparente, las plantas expuestas a esta última revelaron un valor de la TAN $23,09 \%$ mayor que las plantas sin cobertura. Por su parte, la cobertura de color azul redujo 9,90\% en valor de la TAN, en relación con las plantas control, sin diferencias significativas entre estos dos tratamientos (Tabla 3).

Si se toma en consideración que la TAN es una medida de la eficiencia promedio de las hojas de la planta o una medida indirecta de la ganancia neta de asimilados por unidad de 
área foliar en una unidad de tiempo (Brown, 1984), los tratamientos con mayor iluminación, como el control y la cobertura transparente, pudieron haber provocado en las plantas una mayor producción de asimilados que las plantas bajo las coberturas de otros colores; sin embargo, en el presente trabajo, las plantas control sin cobertura y con cobertura transparente no mostraron diferencias significativas con los tratamientos con coberturas roja y verde, cuyos materiales tenían mayor grado de opacidad. En el presente estudio, la respuesta de las plantas de $Z$. aethiopica, en cuanto a la TAN, presentó patrones similares a los de la eficiencia en el uso del agua, pues de hecho, ambas variables están relacionadas con el rendimiento en términos de masa seca. La eficiencia en el uso del agua indicó un mayor valor promedio en las plantas expuestas a la cobertura transparente, como ya se discutió. De igual manera, la TAN, en ese tratamiento, también se vio favorecida, no como consecuencia de una mayor área foliar, sino por una mayor eficiencia en el uso del agua.

Distribución de la materia seca: La exposición de las plantas de cala a las coberturas de color modificó su patrón de asignación de materia seca a los diferentes órganos, con diferencias estadísticamente significativas en hojas, en peciolos y en raíces, pero no en flores $(\mathrm{P}<0,01)$. En el caso de la raíz, se encontraron los menores porcentajes de masa seca, cuando las plantas se expusieron a las coberturas de color azul y verde, mientras que los porcentajes más altos se evidenciaron en las plantas control sin cobertura y en aquellas cubiertas con celofán transparente y amarillo. En cuanto a la asignación de masa seca a las hojas, la cobertura transparente indujo la menor acumulación de masa seca en estos órganos, mientras que el valor más alto se presentó cuando las plantas se expusieron a la cobertura azul. Por otro lado, cuando las plantas crecieron bajo las coberturas azul y verde, acumularon mayor porcentaje de materia seca en los pecíolos, que con los demás tratamientos (Figura 1).

Los patrones de asignación de masa seca a los diferentes órganos de la planta de $Z$. aethiopica, se modificaron con la exposición de las plantas a las diferentes coberturas de color, en especial, bajo las coberturas de color azul y verde, puesto que, según reporte de Wilfret et al. (1976), el sombreado causa etiolación de los pedúnculos, lo que facilita su elongación. Por otro lado, la maximización del área foliar relativa a la biomasa (Relación de área foliar - RAF), se logra mediante un aumento de la expansión de la biomasa foliar en el espacio (Björkman, 1981), como sucedió en las plantas colocadas bajo las coberturas azul y verde, que tuvieron mayor acumulación de masa seca en las hojas, en comparación con los demás tratamientos. Otro estudio (Svenson, 1993), en el cual, en plantas de fresa colocadas en materos de colores verde o blanco y expuestas a diferentes grados de sombreado, se encontró que el color del contenedor no tuvo influencia sobre en masa seca de coronas y hojas; sin embargo, un sombreado del $60 \%$ combinado con el color blanco de la matera redujo notablemente el masa seca de los órganos de la parte aérea y de los frutos, en comparación

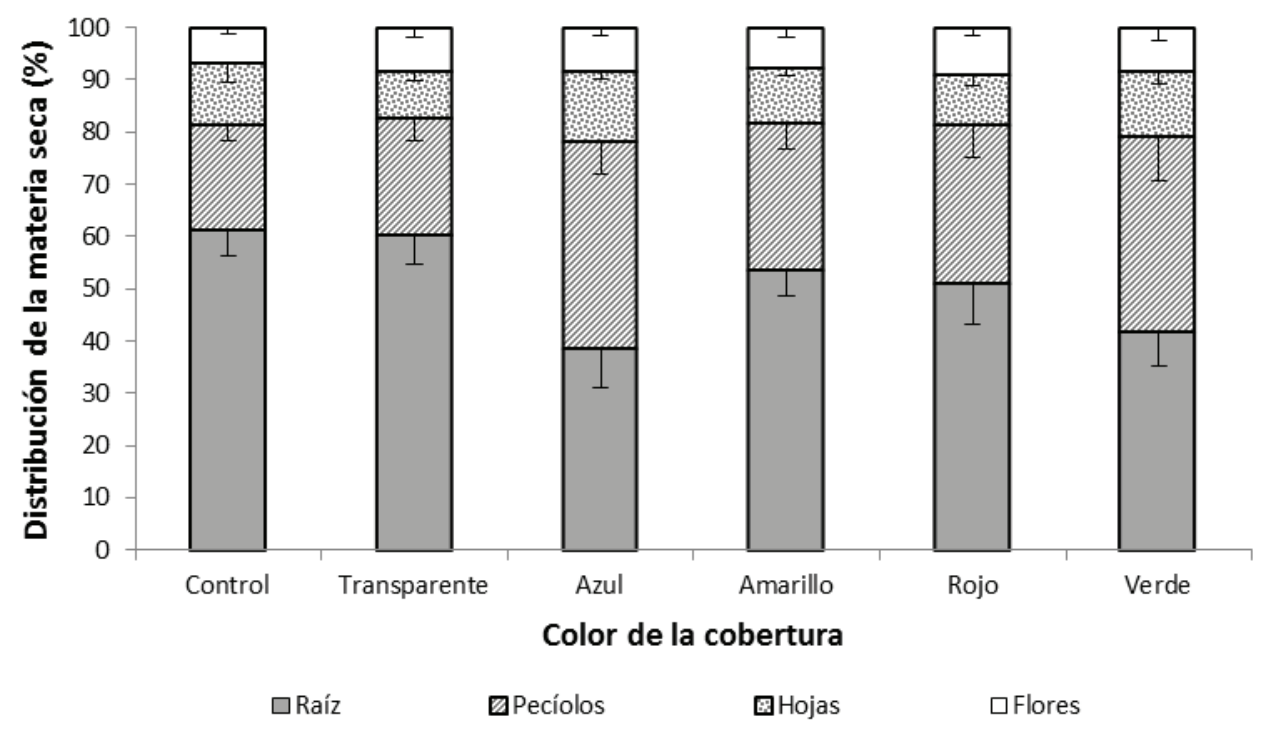

Figura 1. Distribución de la materia seca en calas, Zantedeschia aethiopica (L.), expuestas a coberturas de colores. 
con el mismo nivel de sombreado y color verde de la matera. Por tanto, se podría inferir que las plantas de $Z$. aethiopica presentan gran plasticidad y que a través de ella responden al sombreado y a la calidad de la luz, con modificaciones morfo-anatómicas, que les permiten ajustarse a la oferta ambiental, sin afectar la asignación de masa seca a las flores.

Dado que la luz incidente enriquecida en una franja específica del espectro visible afecta el crecimiento y las características morfológicas de las plantas de cala, es posible mejorar la calidad de las flores, en cuanto a la longitud del pedúnculo, cuando las plantas se exponen a la luz de color verde; no obstante, como se evidenció a través de los resultados del presente trabajo, a pesar que estas plantas se ajustan, hasta cierta medida, a la oferta ambiental en relación con la cantidad de iluminación, muestran mejores respuestas en cuanto al crecimiento, cuando se exponen a alta iluminación. Así que colocar iluminación adicional con focos de luz en el rango del verde, bajo condiciones de plena iluminación, sería una práctica que contribuiría positivamente al mejoramiento del crecimiento y la calidad de flores en estos vegetales.

Agradecimientos: Este estudio, se desarrolló con el apoyo de la Dirección de Investigaciones (DIN) de la Universidad Pedagógica y Tecnológica de Colombia (UPTC), en el marco del plan de trabajo del grupo de investigación Ecofisiología Vegetal, adscrito al programa de Ingeniería Agronómica de la Facultad de Ciencias Agropecuarias de la UPTC. Conflictos de intereses: El manuscrito fue preparado y revisado con la participación de todos los autores, quienes declaramos que no existe ningún conflicto de intereses que ponga en riesgo la validez de los resultados presentados.

\section{BIBLIOGRAFÍA}

1. ALYABYEV, A.JU.; LOSEVA, N.L.; JAKUSHENKOVA, T.P.; RACHIMOVA, G.G.; TRIBUNSKIH, V.I.; ESTRINA, R.I.; PETROV, V.Y. 2002. Comparative effects of blue light and red light on the rates of oxygen metabolism and heat production in wheat seedlings stressed by heat shock. Thermochimica Acta. 394:227-231.

2. BJÖRKMAN, O. 1981. Responses to different quantum flux densities. En: Physiological plant ecology. I. Responses to the physical environment. (Eds. O.L. Lange, P.S. Nobel, C.B. Osmond y H. Ziegler). Springer-Verlag. Encycl. Plant Physiol. New Ser.12A. p.57-107.

3. BROWN, R.H. 1984. Growth of the green plant. En: Physiological basis of crop growth and development. Am. Soc. Agr., Madison. p.153-174.
4. CASIERRA-POSADA, F.; ROJAS B., J. 2009. Efecto de la exposición del semillero a coberturas de colores sobre el desarrollo y productividad del brócoli (Brassica oleracea var. italica). Agr. Col. 27(1):4955.

5. CASIERRA-POSADA, F.; PEÑA-OLMOS, J.E.; ULRICHS, C. 2011. Crecimiento y eficiencia fotoquímica del fotosistema II en plantas de fresa (Fragaria sp.) afectadas por la calidad de la luz: Implicaciones agronómicas. Rev. U.D.C.A Act. \& Div. Cient. 14(2):43-53.

6. CRUZ-CASTILLO, J.G.; TORRES-LIMA, P.A.; ALFAROCHILMALHUA, M.; ALBORES-GONZÁLEZ, M.L.; MURGUÍA-GONZÁLEZ, J. 2008. Lombricompostas y apertura de la espata en poscosecha del alcatraz 'Green Goddess' (Zantedeschia aethiopica (L) K. Spreng) en condiciones tropicales. Rev. Chapingo. Serie Hortic. 14(2):207-212.

7. DE PASCALE, S.; PARADISO, R. 2006. Influencia del régimen térmico y del GA3 sobre Zantedeschia aethiopica L. 3er Congreso Argentino de Floricultura. Libro de resúmenes. La Plata, Argentina. p.21-24.

8. FUKUDA, N.; NISHIMURA, S.; NOGI, M. 2002. Effects of localized light quality from light emitting diodes on geranium peduncle elongation. Acta Hort. 580:151156.

9. FUNNELL, K.A. 1993. Zantedeschia En: De Hertogh, A.; Le Nard, M. The physiology of flower of bulbs. Elseiver. Amsterdam. p.683-704.

10. FUNNELL, K.A. 1994. Calla growers' handbook. Disponible desde Internet en: http://www.callacouncil. org.nz/e_books.asp?id $=$ list\&s $=\& b I D=1 \quad$ (con acceso 15/07/11).

11. HATFIELD, J.L.; SAUER, T.J.; PRUEGER, J.H. 2001. Managing soils to achieve greater water use efficiency: a review. Agr. J. 93:271-280.

12. HEO, J.W.; LEE, C.W.; MURTHY, H.N.; PAEK, K.Y. 2003. Influence of light quality and photoperiod on flowering of Cyclamen persicum Mill. cv. 'Dixie White'. Plant Growth Reg. 40(1):7-10.

13. INOUE, S.; KINOSHITA, T.; TAKEMIYA, A.; DOI, M.; SHIMAZAKI, K. 2008. Leaf positioning of Arabidopsis in response to blue light. Molecular Plant. 1(1):1526. 
14. HUNT, R. 1990. Basic growth analysis. Plant growth analysis for beginners. Londres, Unwin Hyman. 112 .

15. JORDI, W.; POT, C.S.; STOOPEN, G.M.; SCHAPENDONK, A.H.C.M. 1994. Effect of light and gibberellic acid on photosynthesis during leaf senescence of alstroemeria cut flowers. Physiol. Plant. 90:293-298.

16. LAWSON, T.; OXBOROUGH, K.; MORISON, J.I.L.; BAKER, N.R. 2002. Responses of photosynthetic electron transport in stomatal guard cells and mesophyll cells in intact leaves to light, $\mathrm{CO}_{2}$, and humidity. Plant Physiol. 128:52-62.

17. LAZO, J.V.; ASCENCIO, J. 2010. Efecto de diferentes calidades de luz sobre el crecimiento de Cyperus rotundus. Bioagro. 22(2):153-158.

18. LIN, C. 2000. Plant blue-light receptors. Trends in Plan Science 5(8):337-342.

19. MALESZEWSKI, S.; KOZŁOWSKA-SZERENOS, B. 1998. Aktualne problemy badań nad aparatami szparkowymi. Wiad. Bot. 42:21-31.

20. MORAES NETO, S.; GONÇALVES, J.; TAKAKI, M.; CENCI, S., GONÇALVES, J. 2000. Crescimento de mudas de algumas espécies arbóreas que ocorrem na mata atlântica, em função do nível de luminosidade. Rev. Arvore. 24(1):35-45.

21. RABIZA-ŚWIDER, J.; SKUTNIK, E. 2004. Effect of light on senescence of cut leaves of Zantedeschia aethiopica Spr. and Hosta Tratt. 'Undulata Erromena'. Folia Hort. Ann. 16(1):161-166.
22. ROBINSON, A.; CLARK, C.; CLEMENS, J. 2000. Using $1 \mathrm{H}$ magnetic resonance imaging and complementary analytical techniques to characterize developmental changes in the Zantedeschia Spreng tuber. J. Exp. Botany. 51:2009-2020.

23. SCHNETTLER M., B.; MERA S., A.; PIHÁN S., R. 2006. Evaluación técnico-económica de la producción de calas de color en la región de la Araucanía, Chile. IDESIA 24(1):17-24.

24. SVENSON, S. 1993. Shading and pot color influence growth and flowering of strawberry firetails. Proc. Fla. State Hort. Soc. 106:286-288.

25. VALLADARES, F.; ARANDA, I.; SÁNCHEZ, D. 2004. La luz como factor ecológico y evolutivo para las plantas y su interacción con el agua. En: Valladares, F. (Ed). Ecología del bosque mediterráneo en un mundo cambiante. Ministerio de Medio Ambiente. EGRAF. Madrid. p.335-369.

26. WANG, H.; GU, M.; CUI, J.; SHI, K.; ZHOU, Y.; YU, J. 2009. Effects of light quality on $\mathrm{CO}_{2}$ assimilation, chlorophyll-fluorescence quenching, expression of Calvin cycle genes and carbohydrate accumulation in Cucumis sativus. J. Photochem. Photobiol. B: Biology. 96:30-37.

27. WILFRET, G.J.; OTTE, J.A.; HARBAUGH, B.K. 1976. Chrysanthemum peduncle elongation and a cost analysis of three production methods. Proc. Fla. State Hort. Soc. 89:316-319.

Recibido: Julio 17 de 2011

Aceptado: Mayo 12 de 2012 